\title{
Estimación del rendimiento potencial de papa en Córdoba, Argentina, a partir de la eficiencia en el uso de la radiación y de la productividad del agua
}

\author{
de la Casa, A.; G. Ovando, L. Bressanini y J. Martínez
}

\begin{abstract}
RESUMEN
Conocer el rendimiento potencial $(R P)$ es necesario para establecer la brecha productiva con respecto al rendimiento que obtiene el productor. Este trabajo utilizó diferentes modelos para estimar $R P$ en papa (Solanum tuberosum L.) de ciclo tardío en Córdoba, Argentina, comparando dos métodos basados en: a) la eficiencia del uso de la radiación $(R P e)$ y b) la eficiencia o productividad del agua, normalizada con el déficit de presión de vapor $\left(R P w^{D}\right)$ y con la evapotranspiración de referencia $\left(R P w^{E T o}\right)$. El $R P e$ para cuatro fechas de plantación entre 2007 y 2012 resultó muy variable $(C V=17,7 \%)$, con un promedio de 9,7 Mg ha-1 de peso seco (PS), disminuyendo entre 1 y $1,4 \%$ del $R P$ por día de atraso de la plantación. El $R P w^{0}$ promedio fue significativamente $(P<0,001)$ menor $\left(8,4 \mathrm{Mg} P S\right.$ ha $\left.^{-1}\right)$, con una variabilidad similar entre años y épocas de plantación ( $C V=17,3 \%)$, y la misma tendencia a disminuir con el retraso de la plantación. El RPW ETo fue ligeramente menor (9,3 Mg PS ha-1) al $R P e$, diferencia que no fue significativa $(\mathrm{P}>0,05)$, y sus resultados fueron menos variables $(\mathrm{CV}=9,7 \%)$.
\end{abstract}

Palabras clave: papa, rendimiento potencial, eficiencia en el uso de la radiación, productividad del agua.

de la Casa, A.; G. Ovando, L. Bressanini and J. Martínez, 2014. Yield potential estimation of potato in Córdoba, Argentina by radiation use efficiency and water productivity. Agriscientia 31 (2): 75-91

\section{SUMMARY}

Knowing the potential yield (RP) of potato crop (Solanum tuberosum L.) is needed to establish the productivity gap compared to yields obtained by producers. This article uses different models to estimate the RP for late potato growing season in Córdoba, Argentina, comparing two methods based on: a) radiation use efficiency $(R P e)$ and b) efficiency or productivity of water (w), which was normalized according to vapor pressure deficit $\left(R P w^{D}\right)$ and the reference crop evapotranspiration (RPWETo). The RPe for four planting dates from 2007 to 2012 was highly variable (CV $=17.7 \%$ ), with a mean value of 9.7 Mg ha- ${ }^{-1}$ of dry weight (PS), showing a gradual trend to decrease with the delay of planting, with a rate between 1 and $1.4 \%$ of $R P$ per day of delay. The 
average $R P w^{D}$ was significantly $(P<0.001)$ lower $\left(8.4 \mathrm{Mg} \mathrm{PS} \mathrm{ha}^{-1}\right)$, with similar variability between years and planting dates ( $C V=17.3 \%)$, and the same trend to decrease was observed as planting was delayed. The RPWETo tended to be only slightly lower (9.3 Mg PS ha ${ }^{-1}$ ) than those obtained with Rpe. This difference was not significant $(P>0.05)$, and its estimates were less variable $(C V=9.7 \%)$.

Keywords: potato, potential yield, radiation use efficiency, water productivity.

A. de la Casa, G. Ovando, L. Bressanini y J. Martínez. Universidad Nacional de Córdoba, Facultad de Ciencias Agropecuarias. CC 509-Ciudad Universitaria, 5000 Córdoba, Argentina. Correspondencia a: delacasa@agro.unc.edu.ar

\section{INTRODUCCIÓN}

El rendimiento potencial de un cultivo se refiere a la productividad que alcanzaría una variedad adaptada al ambiente en el que crece, bajo condiciones nutricionales e hídricas no limitantes, y con plagas y enfermedades controladas de manera eficaz (Evans, 1993). Bajo este contexto potencial y para una población de plantas en particular, el rendimiento de un cultivo es el resultado de la interacción entre el genotipo y el ambiente, expresado por una combinación particular de radiación solar, que determina la tasa de fotosíntesis, y de temperatura del aire que, además de las funciones fisiológicas del crecimiento, regula también la tasa de desarrollo del cultivo (van Ittersum \& Rabbinge, 1997). El rendimiento disminuye con respecto a este potencial teórico en la medida que el conjunto de factores ambientales y tecnológicos no acompañan las necesidades del cultivo, mientras que la disponibilidad de agua es en cantidad, calidad u oportunidad una de las restricciones más habituales que enfrenta la producción agrícola (Kadaja \& Tooming, 2004; Grassini et al., 2009).

Cuando en una región los recursos son restringidos, la cuantificación del rendimiento potencial de un cultivo y de su productividad es esencial para conocer la magnitud de la brecha entre los rendimientos reales de explotación (es decir, los obtenidos por los agricultores) y aquellos que son posibles de alcanzar, tanto para utilizar como referencia tecnológica, evaluar escenarios de cambio climático global, estimar la capacidad futura de producción de alimentos o formular políticas que garanticen la seguridad alimentaria local y mundial (Cassman et al., 2003; Grassini et al., 2011). La escasa información experimental acerca del rendimiento potencial de un cultivo hace difícil dis- poner de cuantificaciones confiables, así como de la disminución productiva ocasionada por el déficit hídrico o nutricional. Por esta razón, es una práctica común estimar el rendimiento, tanto de carácter potencial como efectivo, con el apoyo de modelos de cultivos; en este caso es imprescindible disponer de datos de suelo y series históricas de variables meteorológicas que incluyen radiación solar, temperatura y lluvia (Yang et al., 2004).

El rendimiento potencial de un cultivo se puede estimar a partir de los dos factores determinantes del crecimiento: la tasa de absorción o intercepción de radiación solar y la tasa de agua transpirada por el cultivo (Stöckle et al., 2003). Esto ha derivado en dos alternativas metodológicas para estimar el rendimiento potencial: una basada en la eficiencia en el uso de la radiación (e $\left[\mathrm{g} \mathrm{m}^{-2} \mathrm{MJ}^{-1}\right]$ ) (Monteith, 1977; Sinclair \& Muchow, 1999), considerada como la relación entre la producción de biomasa o rendimiento y la radiación solar fotosintéticamente activa absorbida o interceptada por el cultivo; y otra que considera la eficiencia o productividad en el uso del agua ( $w\left[\mathrm{~g} \mathrm{~m}^{-2} \mathrm{~mm}^{-1}\right]$ ) (Stanhill, 1986), que corresponde a la pendiente entre la materia seca y la transpiración (Tr) de la canopia en términos acumulativos.

A partir de estos conceptos se establecen los denominados "motores del crecimiento", que los modelos de cultivo utilizan para determinar la productividad agrícola, estructurados sobre la base de relaciones establecidas entre los principales procesos involucrados (tanto la asimilación de $\mathrm{CO}_{2}$ como la transpiración) y el crecimiento, o entre la energía conductora del proceso (radiación solar) y el crecimiento. La cantidad de información producida, el nivel de penetración o difusión y el grado de análisis estructural respecto al uso de $w$, sin em- 
bargo, es sustancialmente menor que la mencionada en la literatura para e. De hecho, la mayoría de los modelos de cultivo más difundidos, como CERES (Jones \& Kiniry, 1986), EPIC (Jones et al., 1991), CROPGRO (Hoogenboom et al., 1992) y STICS (Brisson et al., 2003), presentan algoritmos para estimar la productividad potencial basados en e. Sólo los modelos CropSyst y PARCH (AzamAli et al., 2001) presentan un motor de crecimiento dual que incorpora en paralelo tanto el enfoque de e como de $w$, aunque con mayor énfasis en el primero. Por su parte, el modelo AquaCrop estima la productividad de los cultivos basado sólo en $w$ (Steduto et al., 2009).

Los modelos de cultivo citados previamente, conocidos como mecanísticos, hacen uso de estos conceptos para determinar el rendimiento potencial. La utilización directamente de los motores de crecimiento para estimar el rendimiento potencial de papa, en lugar de recurrir a las rutinas que proveen los modelos de simulación, se justifica ya que, por una parte, configuran procedimientos analíticos más simples y, por la otra, el cálculo queda enteramente bajo control del investigador, con la ventaja de evaluar los resultados conociendo exactamente el procedimiento utilizado.

Mientras la mayor difusión que ha alcanzado el uso de e se sostiene por su comportamiento relativamente más conservativo, los valores de $w$ pueden variar de acuerdo a las condiciones climáticas que determinan la tasa de transpiración y, por lo tanto, se han propuesto distintas alternativas de normalización a fin de comparar y extrapolar evaluaciones de diferentes años y/o lugares (Tanner \& Sinclair, 1983). Con este propósito, dos métodos son comúnmente utilizados para normalizar $w$ : uno que considera el déficit de presión de vapor atmosférica y otro que usa la evapotranspiración de referencia del cultivo (Steduto \& Albrizio, 2005; Steduto et al., 2007).

Con respecto al cultivo de papa, la especie hortícola más importante de la Argentina, Caldiz et al. (2001) desarrollaron una zonificación agroecológica de todo su territorio y obtuvieron el potencial de rendimiento a partir de un modelo de simulación de cultivo que aplica el concepto de e para estimar el crecimiento. Sus resultados presentan un rango amplio entre 10 y $20 \mathrm{Mg} \mathrm{ha}^{-1}$ de materia seca (PS), considerando diferentes regiones y temporadas de producción. Estas determinaciones se realizaron a partir de información climática mensual, lo que morigera en buena medida la variabilidad natural de los registros meteorológicos. Asimismo, los escasos registros de radiación solar imponen utilizar estimaciones de esta variable obtenidas a partir de información meteorológica complementaria, a los efectos de implementar estudios de alcance regional (de la Casa et al., 2003), lo que redunda también en evaluaciones menos precisas. Por su parte, no se encontraron antecedentes en la región respecto a la determinación del rendimiento potencial a partir de la productividad del agua.

La superficie dedicada al cultivo de papa en la Argentina tuvo una evolución contrapuesta en las principales provincias productoras (Mosciaro, 2004). Mientras el área plantada en Buenos Aires disminuyó de $62 \%$ del total nacional a $38 \%$, desde la década de 1980 hacia finales de 1990, la participación de Córdoba aumentó en igual periodo de $13 \%$ a $45 \%$. En Córdoba, Argentina, la papa presenta dos épocas de cultivo al año: la temprana (entre agosto y diciembre) y la tardía (entre febrero y junio), cada una con sus propias limitaciones climáticas (de la Casa et al., 1999). Mientras los cultivos de papa temprana presentan restricciones a la plantación por condiciones de suelo muy frío a la salida del invierno, en los tardíos, tanto el excesivo calor como la persistencia de las lluvias en verano producen frecuentemente atrasos en la fecha de plantación. De acuerdo a resultados experimentales en la región, los lotes de papa tardía muestran una importante heterogeneidad del rendimiento, y una merma productiva relacionada con el atraso de la plantación en la medida que el cultivo no puede extender su ciclo hacia el invierno (de la Casa et al., 2012).

Los objetivos de este trabajo fueron:

- Evaluar el rendimiento potencial de papa de ciclo tardío u otoñal en el cinturón verde de la ciudad de Córdoba a partir de registros meteorológicos diarios, considerando en particular el efecto que un retraso en la fecha de implantación origina sobre la productividad del cultivo.

- Efectuar una comparación de las dos vías alternativas para calcular la productividad a tasa potencial, con el propósito de analizar la condición de complementariedad de e y $w$. En este sentido, a los efectos de normalizar $w$ en términos climáticos se evalúan resultados obtenidos tanto a partir del déficit de presión de vapor como de la evapotranspiración de referencia.

\section{MATERIALES Y MÉTODOS}

\section{Datos}

La información meteorológica se obtuvo de los registros de una estación automática ubicada en proximidad al cinturón verde de la ciudad de Córdoba (3126'17" S, 6411'35" W, altitud de 390 m s 
n m). Una evaluación previa puso de manifiesto la pertinencia de estos datos para su extrapolación al cinturón verde con fines agrometeorológicos (de la Casa \& Nasello, 2006).

Cada hora o fracción de hora el adquisidor realiza el registro de radiación solar, temperatura, humedad relativa del aire y velocidad del viento a 2 $\mathrm{m}$ de altura, originando series de tiempo para cada variable que se encuentran disponibles desde 2003, año en que se instaló la estación, hasta la fecha. Los registros originales fueron procesados para obtener el valor diario de radiación solar (RS), temperatura máxima $\left(T_{\text {max }}\right)$, temperatura mínima $\left(T_{\text {min }}\right)$, humedad relativa máxima $\left(H R_{\text {max }}\right)$, humedad relativa mínima $\left(H R_{\min }\right)$ y velocidad del viento $\left(u_{2}\right)$. Como resultado del ajuste inicial de la estación, los datos meteorológicos presentan mayor discontinuidad al comienzo de la serie. Por esta razón, y para evitar el uso de metodologías de relleno de datos, el análisis se implementó sólo entre 2007 y 2012. Algún dato faltante ocasional se completó durante estos años realizando el promedio con los registros inmediatos anterior y posterior de la serie.

\section{Información agrometeorológica}

Los datos de RS, $T_{\text {max }}, T_{\text {min }}, H R_{\text {max }}, H R_{\text {min }}$ y u 2 se utilizaron para estimar la evapotranspiración potencial de referencia diaria $\left(E T_{0}\right)$ por el método de Penman-Monteith (Allen et al., 1998), mediante el procedimiento ETCalc (Raes, 2009), según la siguiente ecuación:

$$
E T_{o}=\frac{0,408 \Delta\left(R_{n}-G\right)+\gamma \frac{900}{T+237} u_{2}\left(e_{s}-e_{a}\right)}{\Delta+\gamma\left(1+0,34 u_{2}\right)} \text { [Ec. 1] }
$$

siendo $E T_{0}$ la evapotranspiración de referencia $\left[\mathrm{mm} \mathrm{dí}^{-1}\right] ; \mathrm{R}_{\mathrm{n}}$ la radiación neta en la superficie del cultivo [MJ m-2 día-1], que fue estimada a partir de RS, considerando un coeficiente de reflexión de $0,23, T_{\max } y T_{\text {min }}$ para calcular la temperatura media diaria a $2 \mathrm{~m}$ de altura $(\mathrm{T})\left[{ }^{\circ} \mathrm{C}\right]$, y la tensión de vapor actual $\left(\mathrm{e}_{\mathrm{a}}\right)$ [kPa]; $\mathrm{G}$ es la densidad del flujo de calor del suelo [MJ $\mathrm{m}^{-2}$ día-1], que asumió un valor nulo; $\mathrm{u}_{2}$ es la velocidad del viento media diaria a $2 \mathrm{~m}$ de altura [ $\left.\mathrm{m} \mathrm{s}^{-1}\right]$; $e_{s}$ es la presión de vapor de saturación a la temperatura T [kPa]; $e_{s}-e_{a}$ o DPV el déficit de presión de vapor [kPa]; $\Delta$ es la pendiente de la curva de saturación en $\mathrm{T}\left[\mathrm{kPa}{ }^{\circ} \mathrm{C}^{-1}\right] ; \gamma$ es la constante psicrométrica $\left[\mathrm{kPa}^{\circ} \mathrm{C}^{-1}\right.$ ]; 900 es el valor que surge de considerar una superficie de pasto hipotética de 0,12 m de altura, con un coeficiente de reflexión de 0,23 y una resistencia fija de la canopia de $70 \mathrm{~s} \mathrm{~m}^{-1}$.

Los valores diarios de tensión de vapor de satu- ración $\left(e_{s}(T)\right)$, tensión de vapor actual $\left(e_{a}\right)$ y déficit de presión de vapor (DPV) se obtuvieron a partir de $H R_{\text {max }}$ y $\mathrm{HR}_{\text {min }}$ empleando, respectivamente, las siguientes expresiones (Allen et al., 1998):

$e_{s}(T)=0,6108 \times \exp \left[\frac{17,27 \times T}{T+237,3}\right]$

[Ec. 2]

$e_{a}=\frac{e_{s}\left(T_{\min }\right) \frac{H R_{\max }}{100}+e_{s}\left(T_{\max }\right) \frac{H R_{\min }}{100}}{2}$

$D P V=e_{s}-e_{a}=\frac{e_{s}\left(T_{\min }\right)+e_{s}\left(T_{\max }\right)}{2}-e_{a}$

siendo e la tensión de vapor actual $[\mathrm{kPa}] ; \mathrm{e}_{\mathrm{s}}\left(\mathrm{T}_{\min }\right)$ la presión de saturación del vapor a la temperatura mínima diaria $[\mathrm{kPa}] ; \mathrm{e}_{\mathrm{s}}\left(\mathrm{T}_{\max }\right)$ la presión de saturación del vapor a la temperatura máxima diaria [kPa]; $\mathrm{HR}_{\max }$ la humedad relativa máxima diaria [\%]; y $\mathrm{HR}_{\min }$ la humedad relativa mínima diaria [\%].

\section{Cobertura del follaje potencial (CC)}

La cobertura del follaje es un parámetro relevante que representa en términos biofísicos la capacidad de un cultivo para interceptar la radiación solar, como así también para discriminar la evapotranspiración entre la cantidad de agua transpirada (Tr) por un cultivo respecto a la que se evapora del suelo. A fin de obtener el valor de CC que exprese un valor potencial de crecimiento, se empleó el procedimiento propuesto por Steduto et al. (2009), que representa el desarrollo de la cobertura potencial (sin restricciones) por medio del acople de tres funciones exponenciales. La primera de ellas es:

$$
C C=C C_{0} \exp ^{C G C \times G D D} \quad \text { [EC. 5] }
$$

donde CC es la cobertura de la canopia en función del tiempo evaluado según los grados días de crecimiento (GDD) y se expresa como porcentaje cubierto del suelo por el follaje; $\mathrm{CC}_{0}$ es el tamaño inicial de la canopia $(G D D=0)$ en fracción; CGC es el coeficiente de crecimiento de la canopia en fracción por grado día, que constituye un valor constante para un cultivo bajo condiciones de crecimiento óptimas y es modulado por efecto del estrés hídrico.

Esta curva representa el crecimiento exponencial del cultivo durante el primer tramo después de la emergencia, cuando el crecimiento de CC es proporcional al tamaño ya existente. En la medida que las plantas comienzan a sombrearse unas 
a otras el desarrollo progresa de acuerdo a la segunda etapa, en la que CC sigue una tasa de decrecimiento exponencial, de acuerdo a la siguiente expresión:

$C C=C C_{x}-\left(C C_{x}-C C_{0}\right) \times \exp ^{-C G C \times G D D}$

donde CCx es la cobertura máxima para condiciones óptimas de crecimiento, que se estableció en $98 \%$, en razón de ser el máximo valor observado en distintos ensayos a campo (de la Casa et al., 2011, 2013).

A medida que el cultivo se aproxima a la madurez fisiológica, CC comienza a manifestar una etapa de declinación producto de la senescencia foliar generalizada. El modelo describe esta tercera etapa de desarrollo del canopeo de acuerdo a la siguiente expresión:

$$
C C=C C_{x}\left[1-0,05\left(\exp ^{\frac{C D C}{C C_{x}} \times G D D_{s}}-1\right)\right]
$$

donde CDC es el coeficiente de declinación de la canopia (en fracción de reducción por grado día), y $\mathrm{GDD}_{\mathrm{s}}$ es el tiempo térmico desde el comienzo de la senescencia.

Los valores que se adoptaron para representar el desarrollo de la cobertura de follaje potencial, de acuerdo a la fecha de plantación, se indican en la Tabla 1 (de la Casa et al., 2013).

Tabla 1. Coeficientes utilizados para representar el desarrollo de la cobertura de follaje de papa de ciclo tardío de acuerdo a la fecha de plantación en Córdoba, Argentina.

\begin{tabular}{ccccc}
\hline \multirow{2}{*}{ Coeficiente } & \multicolumn{4}{c}{ Fecha de plantación } \\
\cline { 2 - 5 } & $1 / 02$ & $10 / 02$ & $20 / 02$ & $2 / 03$ \\
\hline CC $_{0}$ & 0,02 & 0,02 & 0,02 & 0,02 \\
CGC $_{C C}$ & 0,016 & 0,015 & 0,014 & 0,013 \\
CC $_{\mathrm{x}}$ & 98 & 98 & 98 & 98 \\
CDC & 0,40 & 0,45 & 0,50 & 0,65 \\
\hline
\end{tabular}

Referencias: $\mathrm{CC}_{0}$ es el tamaño inicial de la canopia (GDD =0); CGC es el coeficiente de crecimiento de la canopia en fracción por grado día; $C_{x}$ es la cobertura máxima para condiciones óptimas de crecimiento; CDC es el coeficiente de declinación de la canopia (en fracción de reducción por grado día).

Los ligeros cambios en los valores de CGC y CDC a medida que la fecha de plantación se retrasa, obedecen a la necesidad de representar condiciones bioclimáticas relativamente más favorables para el cultivo hacia comienzo del año, lo que acelera la tasa de crecimiento del canopeo, y la mayor tasa de declinación del canopeo a medida que las condiciones térmicas se prolongan hacia el invierno.
El transcurso del tiempo, por su parte, se evaluó con base en el concepto de tiempo térmico para considerar la influencia de la temperatura del aire sobre la tasa de desarrollo del cultivo. El siguiente procedimiento se empleó para calcular los grados días de crecimiento acumulados durante el ciclo desde plantación a cosecha (GDD):

$$
G D D=\sum_{p}^{c} \frac{T_{\max }^{*}+T_{\min }^{*}}{2}-T_{b}
$$

siendo $T_{\max }^{*}$ y $T_{\min }^{*}$ los valores de temperatura máxima y mínima ajustados, respectivamente; $\mathrm{T}_{\mathrm{b}}$ la temperatura base y $T_{\text {sup }}$ la temperatura superior del cultivo, cuyos valores fueron $2{ }^{\circ} \mathrm{C}$ y $31^{\circ} \mathrm{C}$, respectivamente (Griffin et al., 1995), considerando para su cálculo las siguientes restricciones (Raes et al., 2009):

$$
\begin{gathered}
T_{\max }^{*}=T_{\max } \\
\text { Si } T_{\max }>T_{\text {sup }} \text {, entonces } T_{\text {max }}^{*}=T_{\text {sup }} \\
\text { Si } T_{\text {max }}^{*}<T_{b} \text {, entonces } T_{\text {max }}^{*}=T_{b} \\
T_{\text {min }}^{*}=T_{\text {min }} \\
\text { Si } T_{\text {min }}>T_{\text {sup }} \text {, entonces } T_{\text {min }}^{*}=T_{\text {sup }} \\
\text { Si } T_{\text {min }}<T_{b} \text {, entonces } T_{\text {min }}^{*}=T_{b}
\end{gathered}
$$

\section{Cálculo del rendimiento potencial}

La producción de biomasa con base en el concepto de eficiencia en el uso de la radiación, de acuerdo a Monteith (1977), se obtuvo de la siguiente forma:

$$
B_{L}=e I_{\text {PAR }} \quad[\text { EC. 9] }
$$

donde $B_{L}$ corresponde a la producción diaria de biomasa en función de la radiación solar fotosintéticamente activa interceptada [ $\left.\mathrm{g} \mathrm{m}^{-2}\right]$, e es la eficiencia en el uso de la radiación $\left[\mathrm{g} \mathrm{MJ}^{-1}\right]$, I PAR $_{\text {la }}$ cantidad de radiación fotosintéticamente activa interceptada por el cultivo [MJ m² día-1].

Por su parte, la productividad potencial también se determinó a partir de los conceptos de eficiencia en el uso del agua $(w)$, usando las siguientes ecuaciones:

$$
\begin{aligned}
B_{T} & =\frac{w^{D} T r}{D P V} \\
B_{T} & =\frac{w^{E T o} T r}{E T_{o}}
\end{aligned}
$$

donde $\mathrm{B}_{\mathrm{T}}$ es producción de biomasa que depende de la transpiración [ $\mathrm{g} \mathrm{m}^{-2}$ día $^{-1}$ ], Tr es la transpiración real o efectiva [ $\mathrm{kg} \mathrm{m}^{-2}$ día $\left.{ }^{-1}\right], w^{D}$ es eficiencia 
en el uso del agua [ $\left.\mathrm{g} \mathrm{m}^{-2} \mathrm{~mm} \mathrm{~m}^{-1}\right]$ normalizada por DPV $[\mathrm{kPa}], w^{E T o}$ es el coeficiente de transpiración de la biomasa (eficiencia en el uso del agua) [ $\mathrm{g} \mathrm{m}^{-2}$ $\mathrm{kPa} \mathrm{\textrm {m } ^ { - 1 }}$ ] normalizado por $\mathrm{ET}_{0}[\mathrm{~mm}]$.

En el primer caso, para implementar la Ec. 9 se consideró la cobertura del follaje (CC) directamente sucedánea de la fracción de radiación interceptada por el cultivo, en tanto la radiación solar fotosintéticamente activa interceptada $\left(I_{\mathrm{PAR}}\right)$, se obtuvo multiplicando diariamente CC por el 50\% de la RS recibida ( $\mathrm{MJ} \mathrm{m}^{-2}$ día-1), para evaluar sólo la energía que tiene acción fotosintética (Sinclair \& Muchow, 1999). Además, de acuerdo a experiencias realizadas en papa en el cinturón verde de Córdoba, la eficiencia en el uso de la radiación (e) adoptó un valor de 2,9 $\mathrm{g} \mathrm{MJ}^{-1}$ PAR (de la Casa et al., 2011).

Por su parte, la evapotranspiración máxima del cultivo de papa $\left(E T_{\max }\right)$ se calculó del producto entre $\mathrm{ET}_{0}$ y el coeficiente de cultivo (Kc) (Glenn et al., 2011). El valor de Kc se determinó, de acuerdo a Allen \& Pereira (2009), también a partir de CC (fracción), utilizando la siguiente expresión:

$$
K c=\left\{\begin{array}{l}
\text { si } C C<0,4 ; \quad 0,4 \\
\text { si } C C \geq 0,4 ; 1,1-(1-C C)^{1,2}
\end{array}\right.
$$

La transpiración $(\operatorname{Tr})$ se obtuvo diariamente del producto entre $E T_{\max }$ y la cobertura del follaje (CC), en tanto $W^{D}$ adoptó un valor de $6000 \mathrm{~g} \mathrm{~m}^{-2} \mathrm{kPa} \mathrm{m}^{-1}$, el máximo para especies C3 (Stöckle \& Nelson, 2000), y w $w^{\text {ETo }}$ se estableció en $21 \mathrm{~g} \mathrm{~m}^{-2}$, de acuerdo a las determinaciones experimentales realizadas en el cinturón verde a partir de mediciones de materia seca y bajo las condiciones climáticas de 2009 y 2010 (de la Casa et al., 2013).

Por último, para poner de manifiesto la repercusión de las condiciones climáticas sobre la producción de papa, además de la variabilidad interanual se analizó la influencia que el retraso en la fecha de plantación produce sobre su productividad potencial. En este sentido, se estableció el mes de febrero como periodo de plantación viable considerando una implantación cada 10 días a partir del 1 de febrero. La plantación de papa desde el mes de marzo acusa un riesgo importante de no completar el ciclo de cultivo por la ocurrencia de heladas tempranas.

\section{RESULTADOS Y DISCUSIÓN}

\section{Desarrollo potencial de la cobertura del follaje}

La Figura 1 muestra el desarrollo que presenta

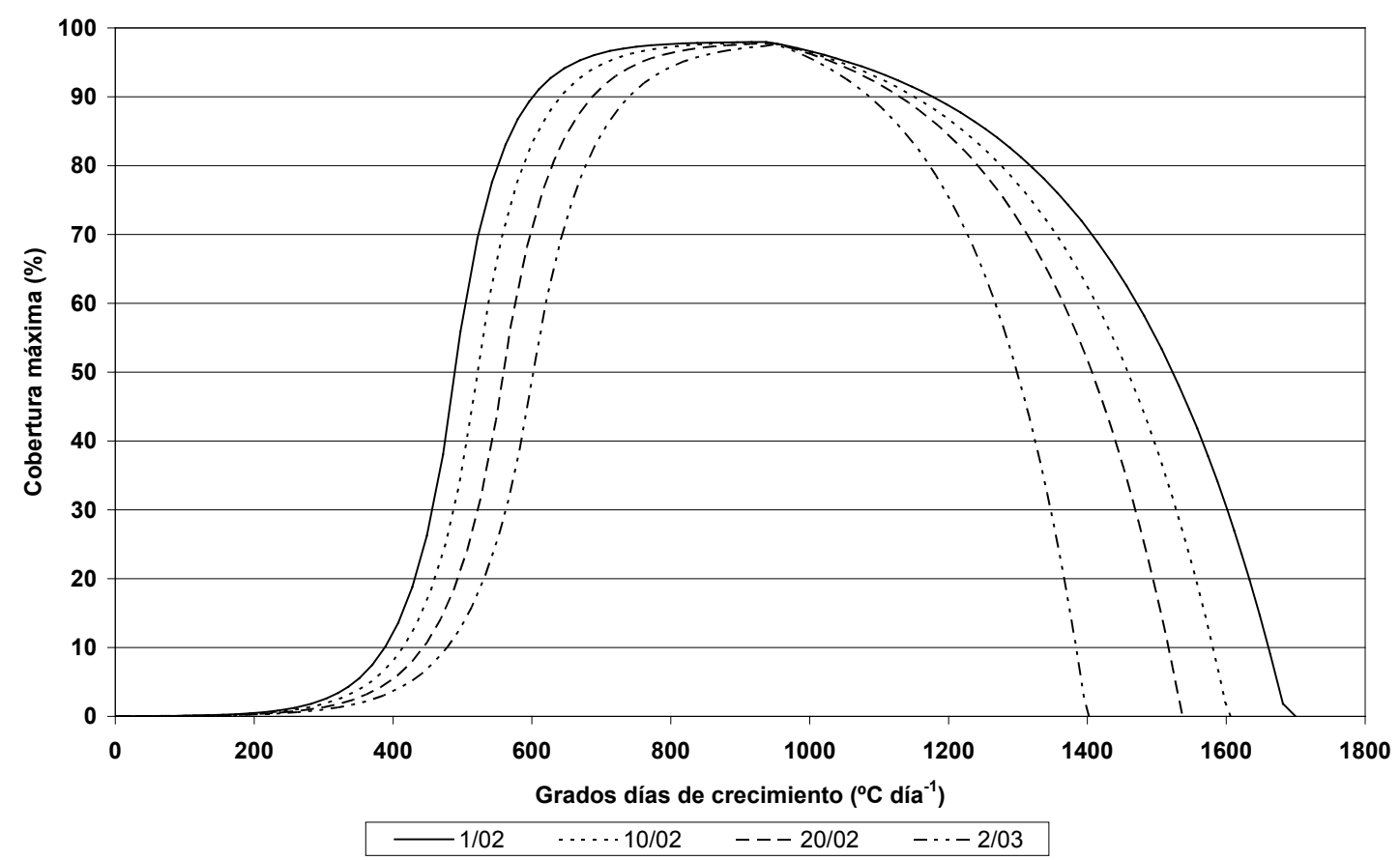

Figura 1. Variación de la cobertura del follaje máximo respecto a los grados días de crecimiento para cada fecha de plantación durante el ciclo 2007 en Córdoba, Argentina. 
la cobertura del follaje de carácter máximo respecto a la escala de tiempo térmico durante el ciclo tardío de papa en Córdoba para cada una de las fechas de plantación consideradas. Para el mismo valor máximo de CCx (98\%), se aprecia el efecto que sobre la cobertura producen tanto la disminución de la tasa de crecimiento de la canopia (CGC) en el tramo ascendente, como el aumento de la tasa de declinación (CDC) en el tramo descendente, a medida que el ciclo de cultivo se desplaza hacia el periodo otoñal cuando la fecha de plantación se atrasa. La extensión del ciclo de papa de acuerdo a las distintas fechas de plantación, en términos tanto del número de días como de los grados días acumulados, se presenta en la Tabla 2.

Tabla 2. Sumas térmicas y días calendarios del cultivo de papa de ciclo tardío de acuerdo a distintas fechas de plantación en Córdoba, Argentina, para el conjunto de años analizado.

\begin{tabular}{ccccc}
\hline & \multicolumn{4}{c}{ Fecha de plantación } \\
\cline { 2 - 5 } Año & 01-Feb & 10-Feb & 20-Feb & 02-Mar \\
\cline { 2 - 5 } & \multicolumn{4}{c}{$\left({ }^{\circ} \mathrm{C}\right.$ días) } \\
\hline 2007 & 1699,7 & 1605,9 & 1536,9 & 1402,2 \\
2008 & 1703,2 & 1616,5 & 1545,7 & 1417,8 \\
2009 & 1698,8 & 1611,2 & 1543,0 & 1410,9 \\
2010 & 1695,9 & 1610,1 & 1541,3 & 1404,5 \\
2011 & 1690,9 & 1617,5 & 1544,1 & 1405,4 \\
2012 & 1686,6 & 1608,8 & 1545,2 & 1411,5 \\
\cline { 2 - 5 } Media & 1695,8 & 1611,7 & 1542,7 & 1408,7 \\
DE & 6,13 & 4,51 & 3,25 & 5,77 \\
\hline & \multicolumn{4}{c}{ Días } \\
2007 & 89 & 89 & 90 & 87 \\
2008 & 85 & 85 & 85 & 79 \\
2009 & 79 & 76 & 75 & 69 \\
2010 & 86 & 83 & 87 & 83 \\
2011 & 85 & 85 & 84 & 78 \\
2012 & 79 & 81 & 80 & 75 \\
\hline Media & 83,8 & 83,2 & 83,5 & 78,5 \\
DE & 4,02 & 4,40 & 5,32 & 6,25 \\
\hline
\end{tabular}

A pesar de producirse una diferencia media de $287^{\circ} \mathrm{C}$ días entre la primera y última fecha de plantación para el conjunto de años, la extensión del ciclo estimada resulta en una disminución de sólo 6 días, que es lo suficientemente pequeña como para deslindar a este factor en la responsabilidad de las diferencias de productividad, particularmente cuando se considera la plantación durante el mes de febrero.

Kooman et al. (1996b) establecieron que la variación en la longitud del ciclo de cultivo, asociada con la temperatura y la duración del día, resultó el factor más importante para explicar diferencias en la producción de materia seca comparando dis- tintos cultivares (incluyendo Spunta) y regiones de producción. El cultivo de papa tardío se encuentra acotado térmicamente en Córdoba, ya que las temperaturas se presentan progresivamente más bajas a medida que el ciclo avanza hacia el otoño e, incluso, alcanzan niveles de congelamiento en algunos años. El ciclo de producción de papa otoñal en Túnez presenta una condición climática similar donde, además de la restricción por las heladas al final del ciclo, la plantación debe demorarse hasta que la temperatura sea inferior a $30^{\circ} \mathrm{C}$ para permitir la producción (Kooman et al., 1996a). Esta limitación justifica considerar una menor suma térmica para las fechas de plantación más tardías. Disminuciones similares en las sumas térmicas fueron observadas por Worthington \& Hutchinson (2006) y Khan et al. (2011), quienes obtuvieron diferentes sumas térmicas para distintas fechas de plantación, con valores más reducidos a medida que las fechas se aproximaron a períodos más fríos. Por otra parte, la reducción del ciclo a medida que el fotoperíodo disminuye en otoño (Haverkort, 1990; Kooman et al., 1996b), parece indicar para la variedad Spunta una respuesta fotoperiódica de días cortos que también puede justificar la menor acumulación térmica.

\section{Rendimiento potencial de papa estimado a partir de la eficiencia en el uso de la radiación}

La radiación solar que se recibe diariamente presenta un comportamiento de notable variabilidad. La Figura 2 muestra los registros observados en los años 2007 y 2011, tomados a modo de ejemplo, durante el ciclo tardío u otoñal de producción de papa en el cinturón verde de Córdoba.

En ambos años la radiación solar muestra una importante variación diaria, asociada con el estado de nubosidad y transparencia atmosférica que son propias de las condiciones meteorológicas existentes. Asimismo, la Fig. 2 expresa también la importante declinación de la energía recibida en términos estacionales, producto del aumento que experimentan el ángulo cenital solar y la disminución de la duración del día para esta latitud, a medida que el tiempo transcurre hacia el solsticio de invierno. Además, en la figura también se ponen de manifiesto diferencias interanuales, resultando a simple vista los valores del año 2011 mayores a los de 2007. Los resultados de la Tabla 3 confirman diferencias estadísticamente significativas entre años para los valores de radiación solar registrados en el mes de marzo $(p<0,05)$ y para el periodo completo entre febrero y mayo ( $p<0,01)$, comportamiento que está asociado a la dinámica 
Tabla 3. Comparación de la radiación solar recibida (RS) entre los años 2007 y 2011 en Córdoba, y estadísticos para evaluar su significancia en distintos periodos.

\begin{tabular}{|c|c|c|c|c|c|c|c|}
\hline \multirow{3}{*}{ periodo } & \multicolumn{4}{|c|}{$\mathrm{RS}\left(\mathrm{MJ} \mathrm{m}^{-2} \mathrm{dí}^{-1}\right)$} & \multirow{3}{*}{ gl } & \multirow{3}{*}{ valor de $t$} & \multirow{3}{*}{$P$} \\
\hline & \multicolumn{2}{|c|}{2007} & \multicolumn{2}{|c|}{2011} & & & \\
\hline & Media & $\mathrm{DE}$ & media & $\mathrm{DE}$ & & & \\
\hline febrero & 17,3 & 7,2 & 19,7 & 6,8 & 54 & $-1,268$ & 0,2104 \\
\hline marzo & 15,0 & 6,9 & 18,5 & 5,7 & 60 & $-2,189$ & 0,0325 \\
\hline abril & 13,1 & 5,2 & 14,8 & 3,7 & 58 & $-1,455$ & 0,1510 \\
\hline mayo & 9,6 & 3,7 & 10,7 & 3,5 & 60 & $-1,178$ & 0,2434 \\
\hline febrero-mayo & 13,7 & 6,5 & 15,8 & 6,1 & 120 & $-2,662$ & 0,0083 \\
\hline
\end{tabular}

Valores de t en cursiva son significativos $(P<0,05)$

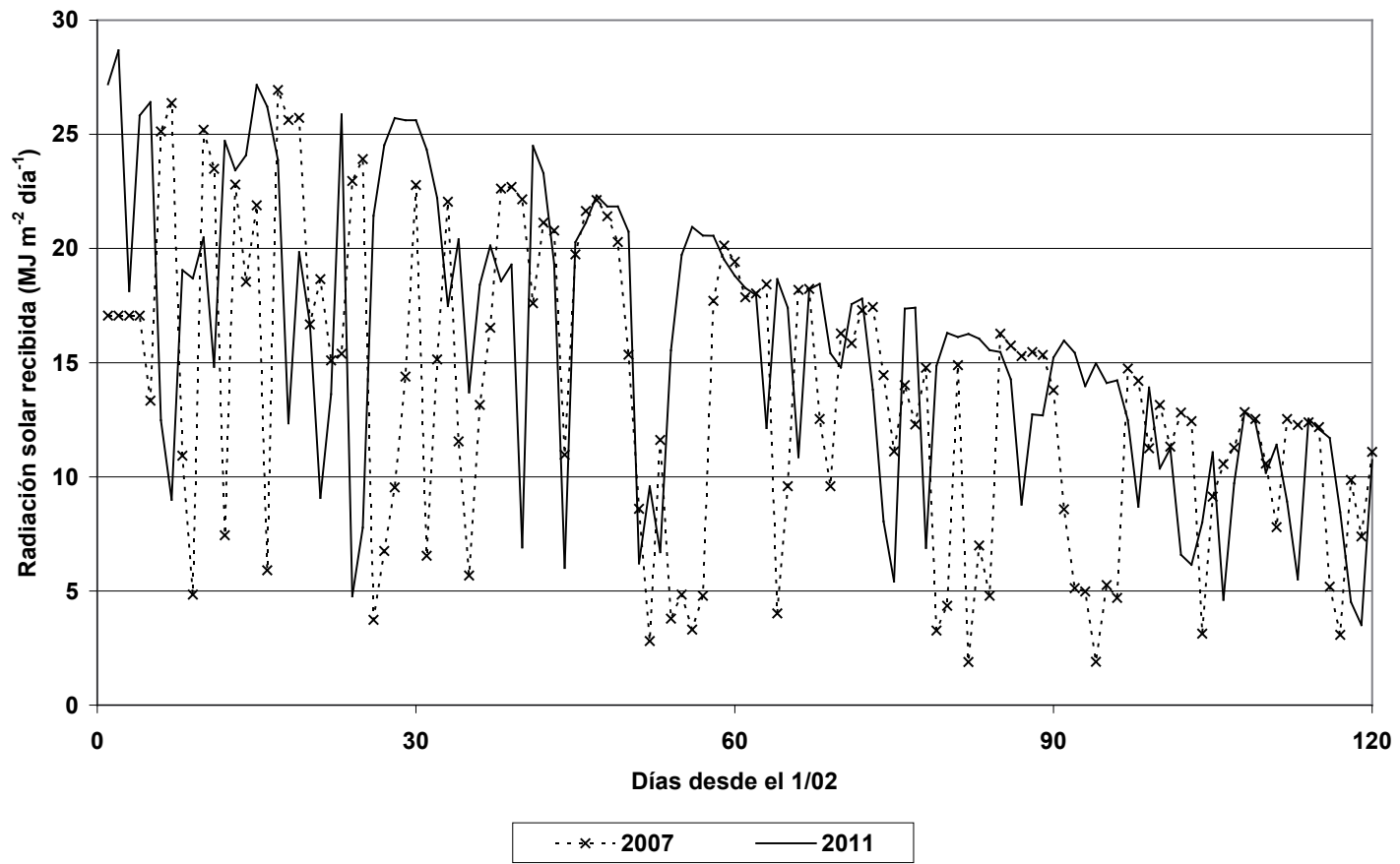

Figura 2. Variación diaria de la radiación solar recibida en el cinturón verde de Córdoba, Argentina, durante el ciclo tardío de papa en 2007 y 2011.

propia de las condiciones meteorológicas de cada año.

A partir de los valores de radiación solar observados y la condición de cobertura del follaje máxima estimada, el rendimiento potencial de papa se determinó para las 4 fechas de plantación aplicando el concepto de eficiencia en el uso de la radiación (e), con los resultados que presenta la Figura 3.

Hasta la segunda fecha de plantación (10/02) las estimaciones de rendimiento están comprendidas dentro del rango entre 10 y $20 \mathrm{Mg} \mathrm{PS} \mathrm{ha}^{-1}$, que Caldiz et al. (2001) presentan para la Argentina a partir del modelo LINTUL-POTATO (Kooman \& Haverkort, 1995), si bien más próximas al valor inferior del intervalo antes mencionado como consecuencia del ciclo más corto. Los valores de rendimiento potencial obtenidos en este estudio también concuerdan con los estimados por Stol et al. (1991) y van Keulen \& Stol (1995). No obstante, Pereira et al. (2008) calculan para el estado de San Pablo, Brasil, valores de rendimiento potencial más elevados, que para distintos años, lugares y épocas de cultivo varían entre 16,8 a 35,7 Mg PS de tubérculo $\mathrm{ha}^{-1}$, valores muy similares al rango que ellos observaron experimentalmente (17,5 a 39,0 Mg PS ha-1).

Las fechas de implantación más tardías, sin embargo, resultan con valores muy por debajo del límite inferior y muestran una tendencia a disminuir de manera progresiva con el atraso de la plantación. A los efectos de evaluar la tasa de disminución de la productividad supeditada al atraso de la plantación, cada año estos valores se expresaron 


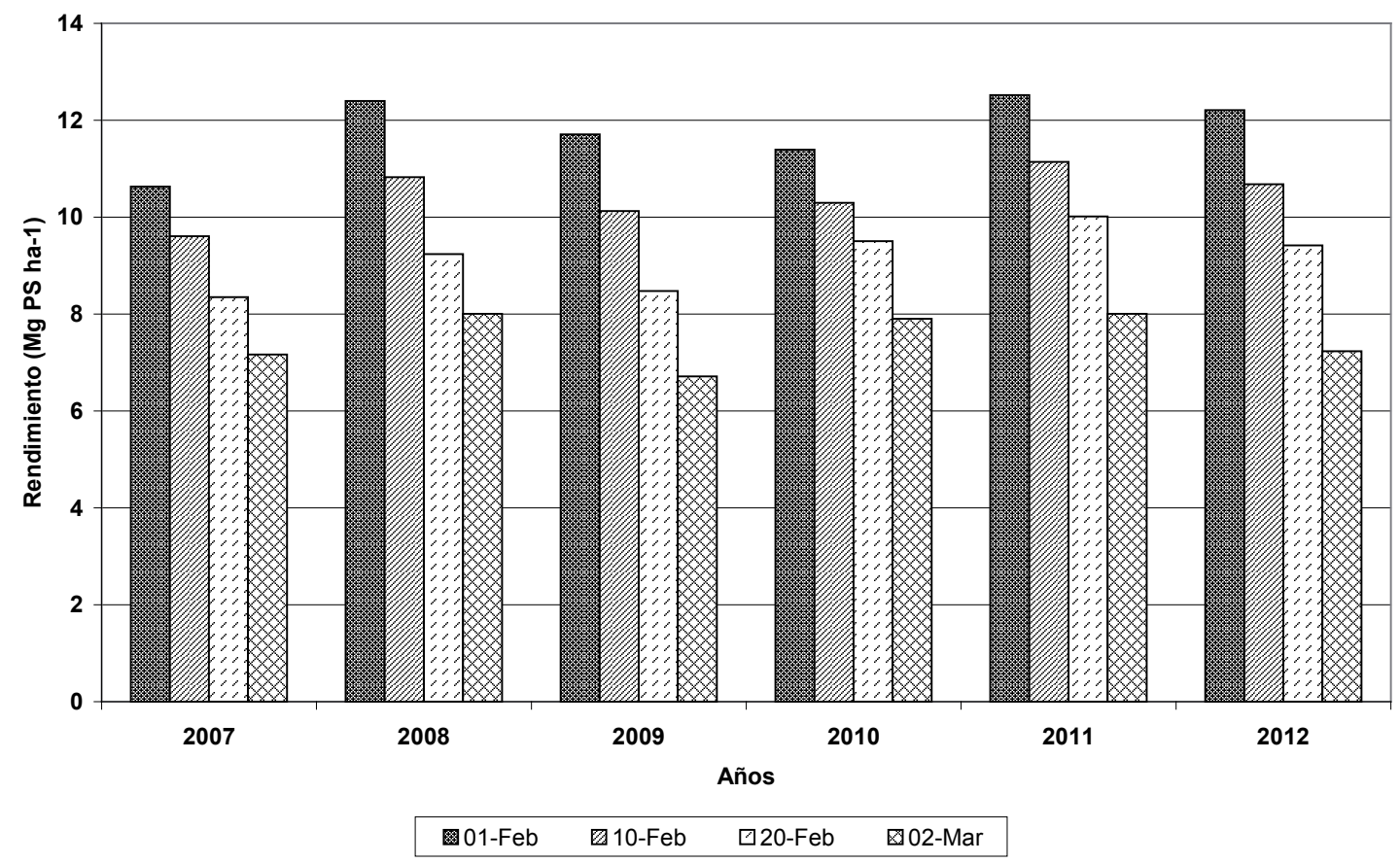

Figura 3. Rendimiento potencial de papa (peso seco (PS)) estimado a partir de la Ec. 9 para distintas fechas de plantación tardías en el cinturón verde de Córdoba, Argentina, entre 2007 y 2012.

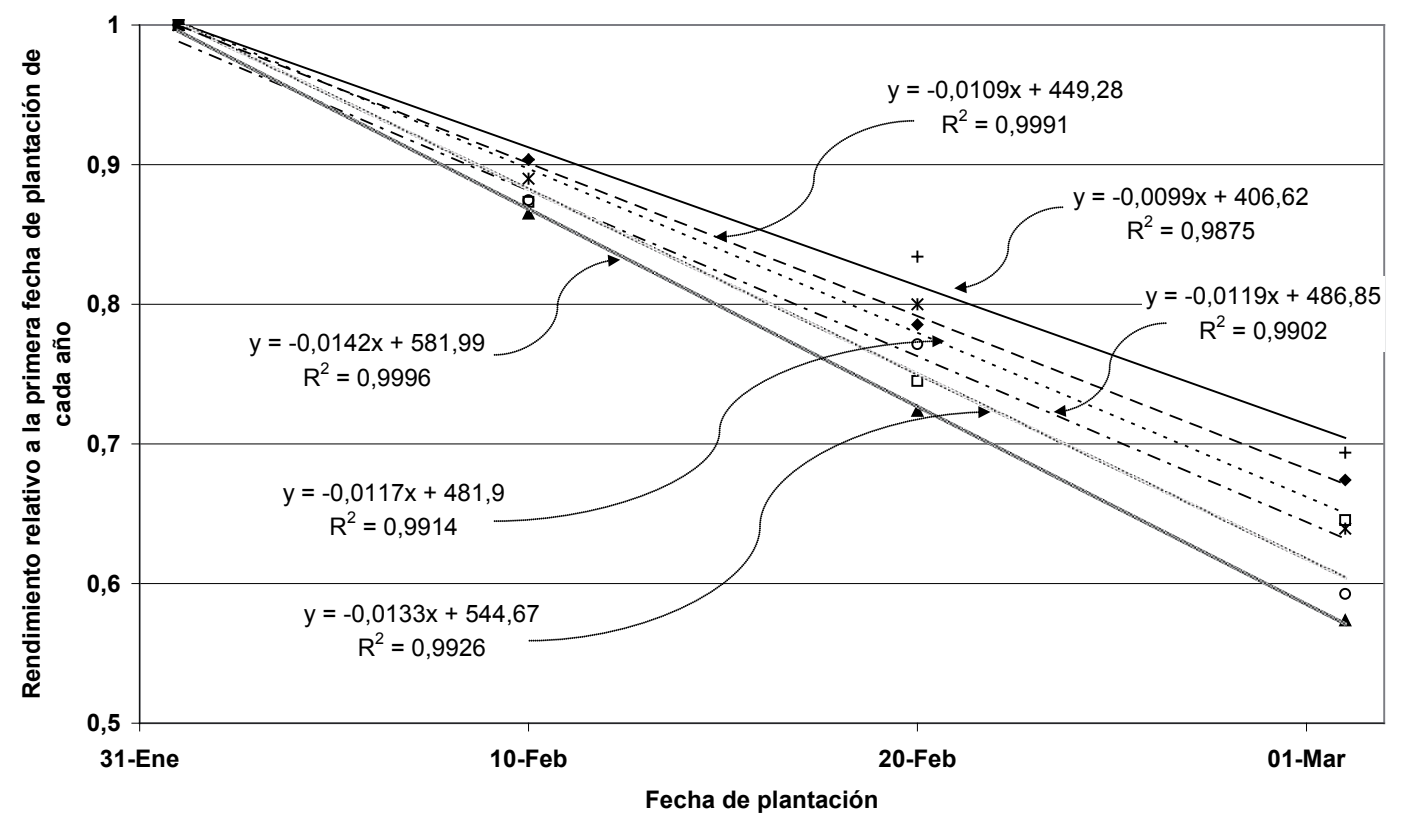

$\begin{array}{rlllll}-2007 \quad \square 2008 & \Delta 2009 & +2010 \quad * 2011 \quad \circ 2012\end{array}$

Figura 4. Variación del rendimiento potencial de papa estimado a partir de la eficiencia en el uso de la radiación (e) con respecto a la fecha de plantación para los ciclos de producción entre 2007 y 2012 en el cinturón verde de Córdoba, Argentina. 
de manera relativa con respecto a la primera fecha de plantación, como muestra la Figura 4. Los resultados de correlación y regresión obtenidos verifican una tasa de disminución lineal que, dependiendo del año, presenta un rango entre 1 y $1,4 \%$ por día de atraso de la plantación.

La variación del rendimiento potencial está ligada estrictamente por su naturaleza a las condiciones meteorológicas/climáticas de una región (Kadaja \& Tooming, 2004). Grassini et al. (2009) determinaron que el rendimiento potencial en maíz depende del régimen de radiación/temperatura específico de cada lugar y su interacción con la fenología del cultivo. La información que desarrolla este trabajo refuerza lo anterior, mostrando una tasa de disminución de la producción en función al atraso de la fecha de plantación que es diferente cada año en razón de la particular disponibilidad de radiación solar y temperatura.

\section{Rendimiento potencial de papa a partir de la eficiencia o productividad en el uso del agua}

\section{Productividad del agua normalizada por DPV}

Los datos básicos para estimar el rendimiento potencial de papa sobre la base del principio de eficiencia o productividad del agua, de acuerdo a la Ec. 10, son la evapotranspiración de referencia $\left(E T_{0}\right)$ y el déficit de presión de vapor (DPV). En la Figura 5 se observa la variación diaria que presentan estas variables durante el ciclo otoñal de producción de papa en Córdoba para los años 2007 y 2011.

En un contexto de variación similar al que pre- senta la Figura 2, en la Figura 5 se observa la importante fluctuación que acusa el valor diario de $\mathrm{ET}_{\mathrm{o}}$, en razón directa a la tasa de energía recibida por radiación solar, como se desprende al considerar el modelo de Penman-Monteith (Ec. 3), y de acuerdo a la relación que muestra la Figura 6 para los años 2007 y 2011. Asimismo, las diferencias de las condiciones meteorológicas entre ambos años, para periodos mensuales o del ciclo completo de la papa, se presentan estadísticamente significativas prácticamente en todos los periodos considerados, con la excepción de febrero, como muestra la Tabla 4. La ETo resultó menor en 2007, en correspondencia con la radiación solar más reducida pero, además, asociada significativamente al DPV que también es más bajo, es decir que con aire relativamente más saturado la demanda atmosférica de agua se reduce.

El rendimiento potencial que se obtiene a partir del principio de productividad o eficiencia en el uso del agua $(w)$ se presenta en la Figura 7. Del mismo modo que muestra la Figura 3 y se evalúa de manera gráfica en la Figura 4, la productividad potencial se reduce a una tasa lineal con el atraso de la fecha de plantación, pero los valores son en general más reducidos, con un rango entre 0,7 en el ciclo 2007 y 1,2\% en 2009 por cada día de atraso de la plantación.

La Figura 8 presenta la dispersión entre los valores de rendimiento potencial que se obtienen a partir de $e$ y $w^{D}$, resultando significativa $(P<$ $0,001)$ la diferencia entre la media de ambos procedimientos y menor en el caso de $W^{D}$. Si bien la correlación entre los resultados de ambos métodos cada año es elevada, la gran dispersión del conjunto indica un efecto marcado que ejercen

Tabla 4. Comparación de la evapotranspiración de referencia (ETo) y el déficit de presión de vapor (DPV) entre los años 2007 y 2011 en Córdoba, y estadísticos para evaluar su significancia en distintos periodos.

\begin{tabular}{|c|c|c|c|c|c|c|c|}
\hline \multirow[t]{3}{*}{ periodo } & \multicolumn{2}{|c|}{2007} & \multicolumn{2}{|c|}{2011} & \multirow[t]{3}{*}{ gl } & \multirow[t]{3}{*}{ valor de $\mathrm{t}$} & \multirow[t]{3}{*}{$p$} \\
\hline & \multicolumn{4}{|c|}{$\mathrm{ET}_{0}\left(\mathrm{~mm}\right.$ día $\left.\mathrm{a}^{-1}\right)$} & & & \\
\hline & media & $\mathrm{DE}$ & media & $\mathrm{DE}$ & & & \\
\hline febrero & 4,5 & 1,4 & 4,7 & 1,5 & 54 & $-0,581$ & 0,5640 \\
\hline marzo & 3,5 & 1,4 & 4,6 & 1,1 & 60 & $-3,436$ & 0,0011 \\
\hline abril & 3,1 & 1,0 & 3,9 & 0,8 & 58 & $-3,487$ & 0,0009 \\
\hline mayo & 2,1 & 0,6 & 2,5 & 0,6 & 60 & $-2,398$ & 0,0196 \\
\hline febrero-mayo & 3,2 & 1,4 & 3,9 & 1,4 & 120 & $-3,544$ & 0,0005 \\
\hline \multicolumn{8}{|c|}{$\mathrm{DPV}(\mathrm{kPa})$} \\
\hline febrero & 1,2 & 0,4 & 1,3 & 0,5 & 54 & $-0,443$ & 0,6595 \\
\hline marzo & 0,9 & 0,5 & 1,5 & 0,4 & 60 & $-4,818$ & 0,0000 \\
\hline abril & 1,0 & 0,5 & 1,5 & 0,4 & 58 & $-4,299$ & 0,0001 \\
\hline mayo & 0,7 & 0,3 & 0,9 & 0,3 & 60 & $-2,499$ & 0,0152 \\
\hline febrero-mayo & 0,9 & 0,4 & 1,3 & 0,5 & 120 & $-5,416$ & 0,0000 \\
\hline
\end{tabular}

Valores de $t$ en cursiva son significativos $(P<0,05)$ 

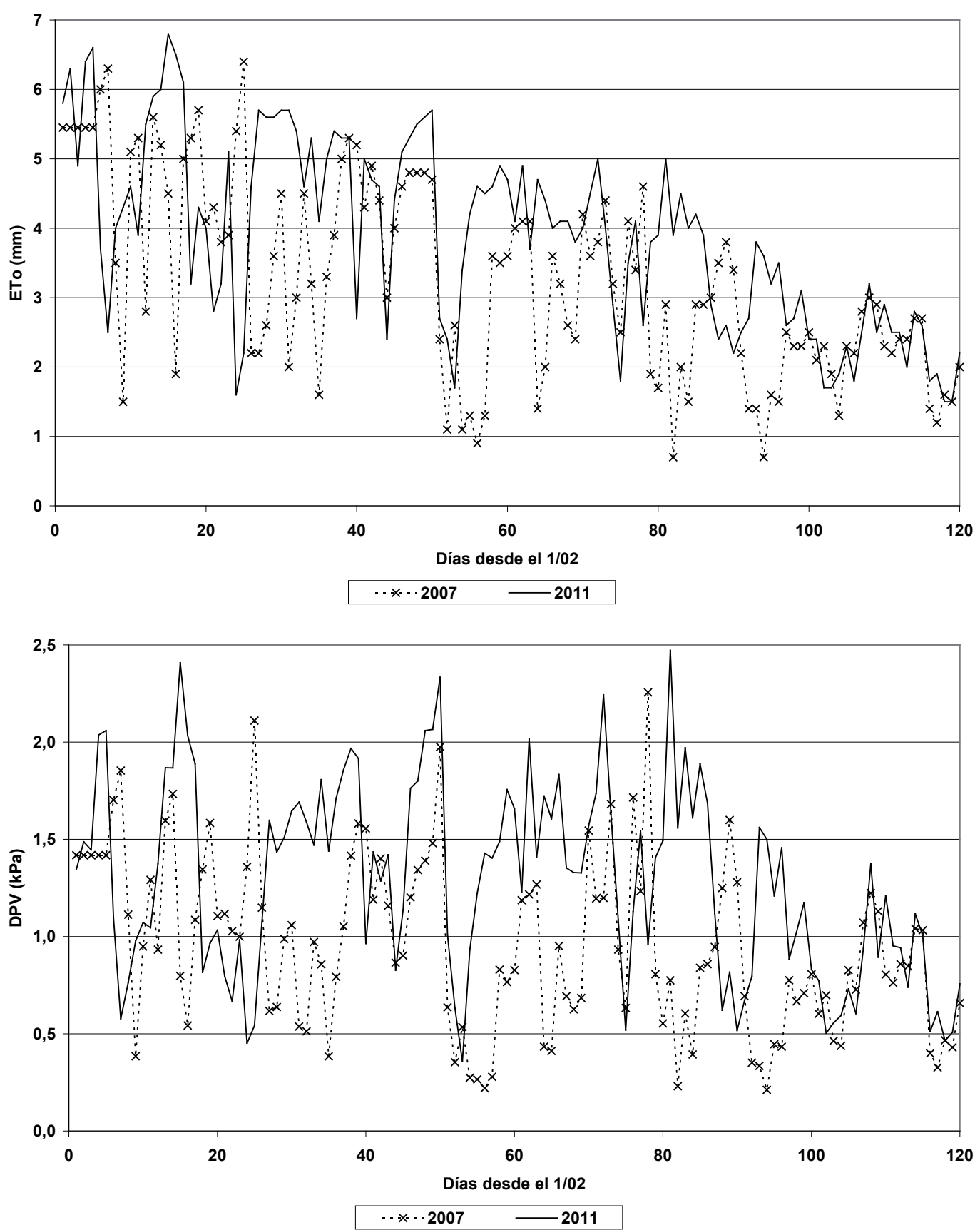

Figura 5. Variación diaria de la evapotranspiración de referencia (ETo) y el déficit de presión de vapor (DPV) desde febrero a mayo de 2007 y 2011 en Córdoba, Argentina.

las condiciones meteorológicas en cada ciclo de producción. Asimismo, las estimaciones obtenidas para las distintas fechas de plantación a través de los años muestran una variabilidad acentuada, con un coeficiente de variación (CV) mayor a $17 \%$ para ambos métodos.
Respecto a la función igualdad, sólo en 2007 se observa que los valores de productividad obtenidos a partir de $w^{D}$ son superiores a los que se determinan con $e$. Las estimaciones del rendimiento potencial que corresponden a 2009, en sentido opuesto, muestran una diferencia igualmente mar- 


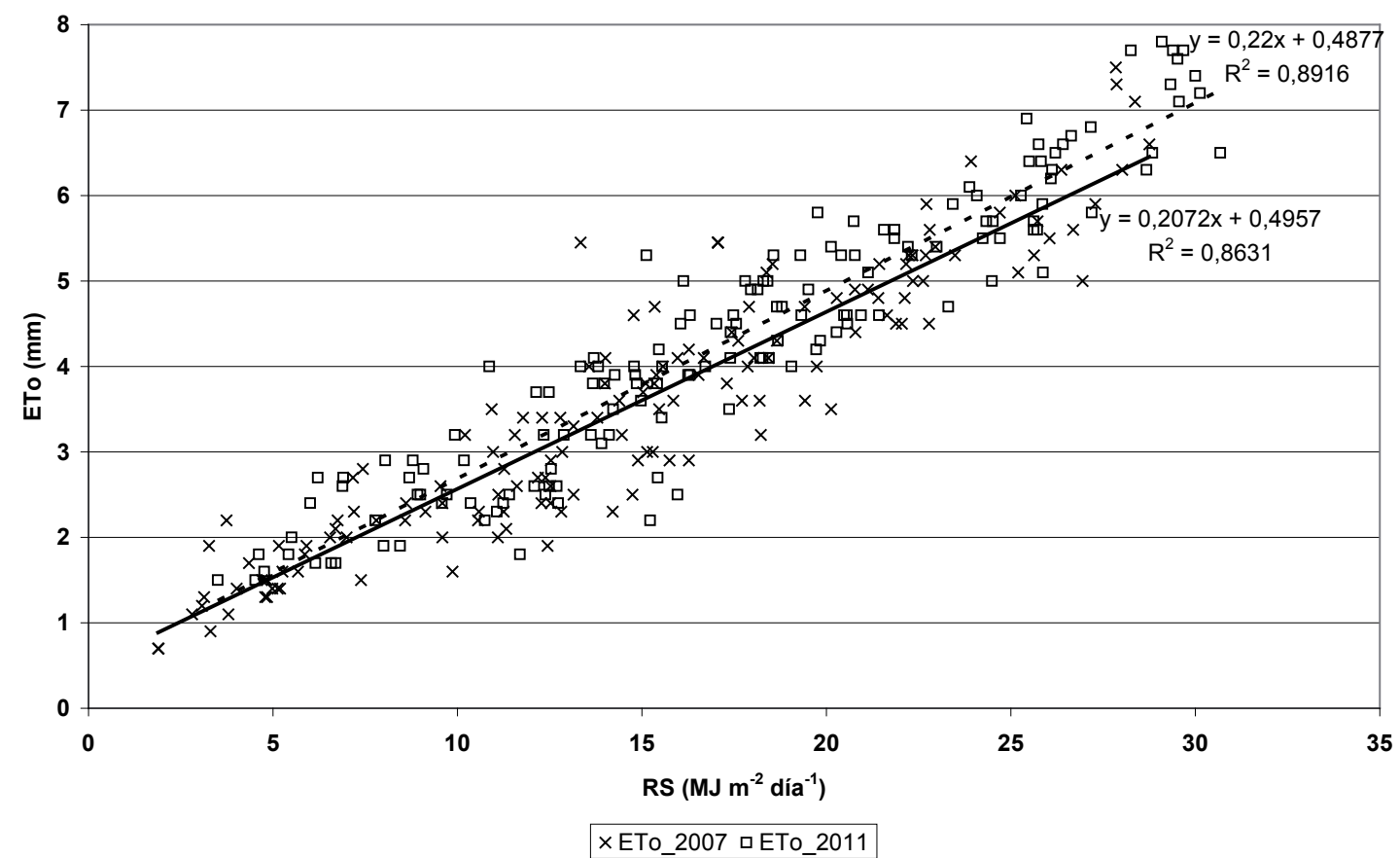

Figura 6. Relación entre los valores diarios de radiación solar (RS) y la evapotranspiración de referencia (ETo) en 2007 y 2011 en Córdoba, Argentina.

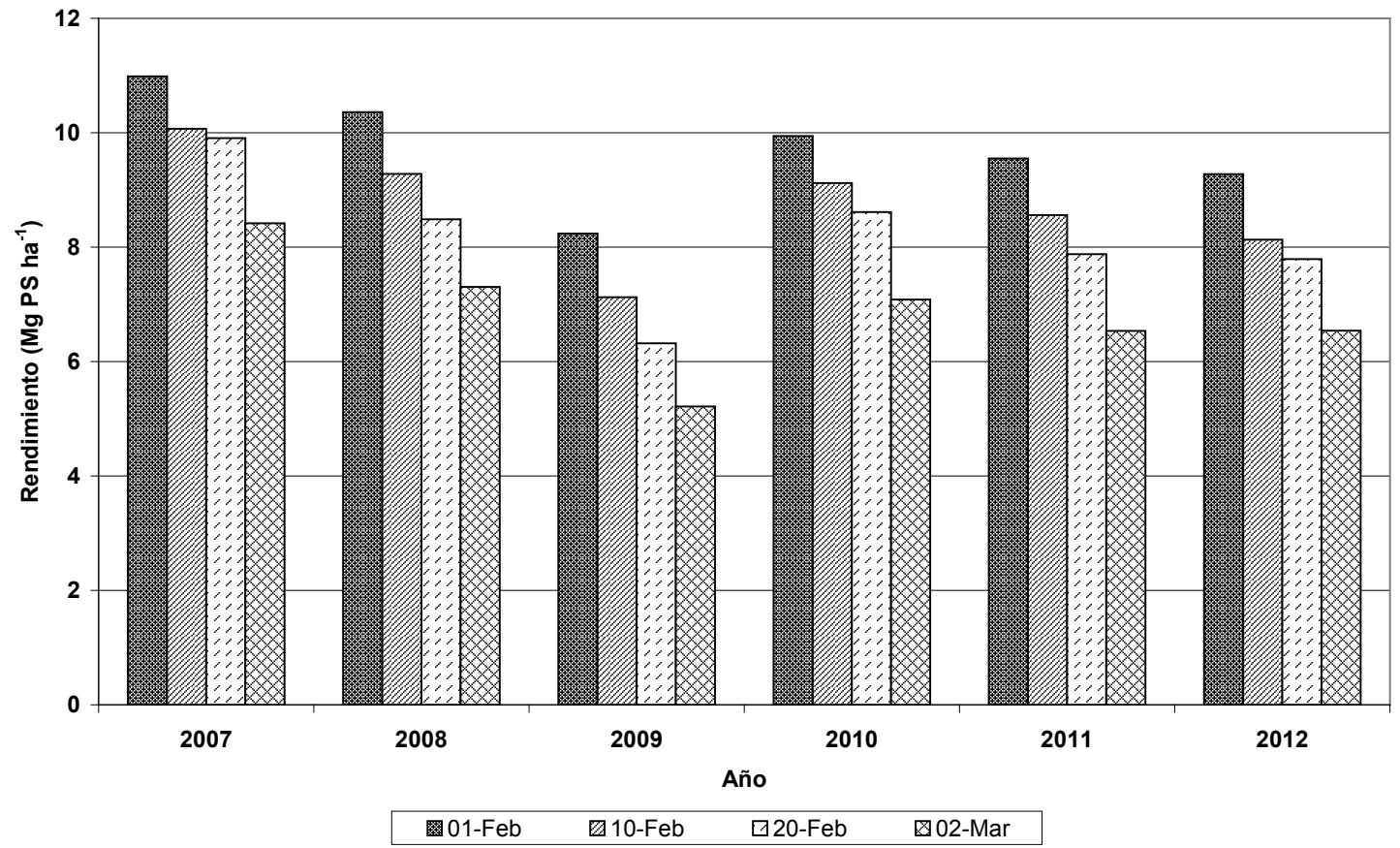

Figura 7. Rendimiento potencial de papa (peso seco (PS)) calculado a partir de la Ec. 10 (con w normalizado de acuerdo al DPV, wD) para las distintas fechas de plantación en los ciclos de producción del cinturón verde de Córdoba, Argentina, entre 2007 y 2012. 


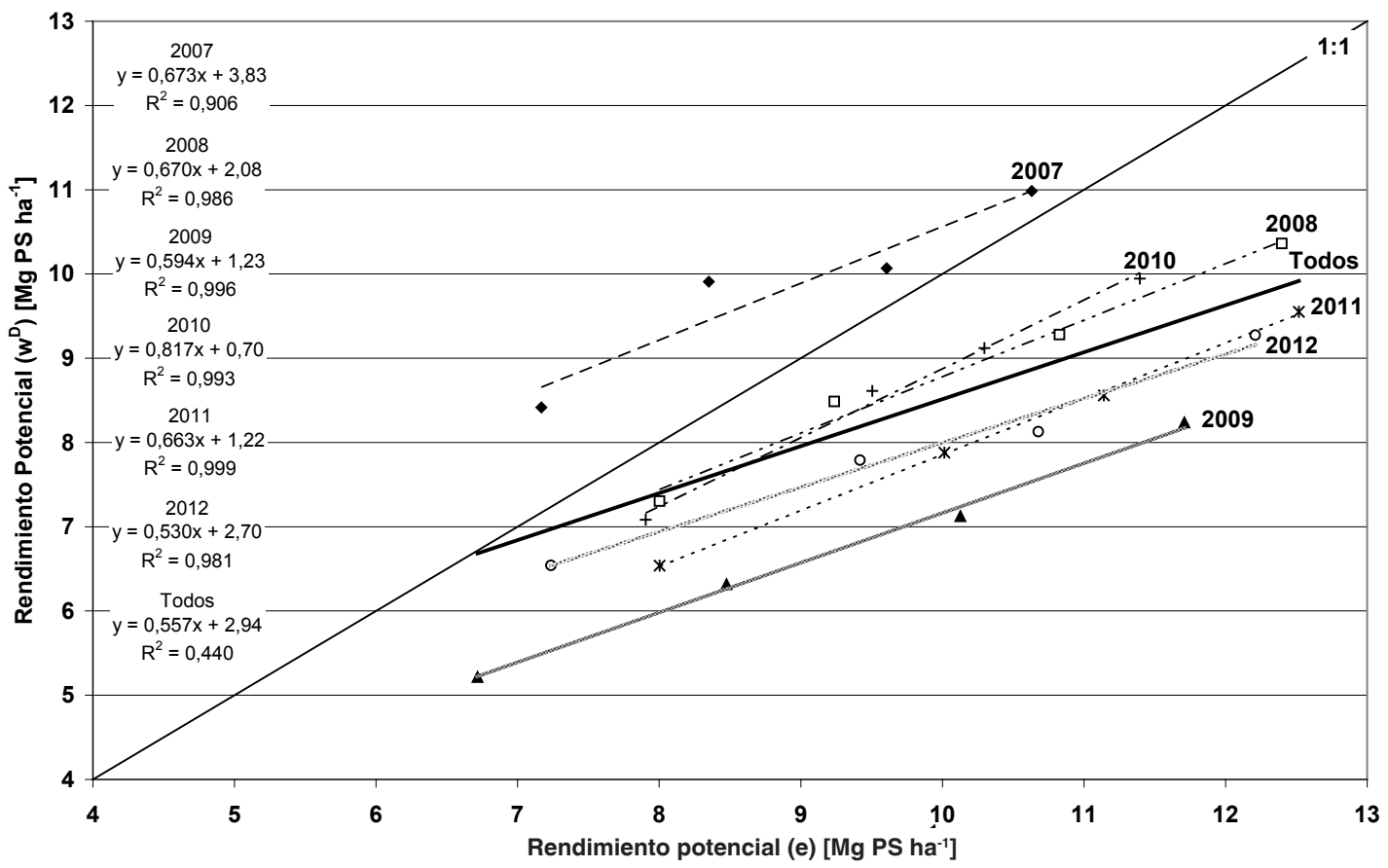

Figura 8. Relación entre los valores de rendimiento potencial obtenidos usando la eficiencia en el uso de la radiación (e) y la eficiencia en el uso del agua normalizada con el déficit de presión de vapor (wD), correspondientes a distintas fechas de plantación para cada año entre 2007 y 2012 en Córdoba, Argentina.

cada entre ambos métodos, pero en este caso $w^{D}$ produce valores notablemente más bajos a los de e. Habiendo determinado en la Figura 6 que la mayor parte de la variabilidad de $\mathrm{ET}_{0}$ obedece a los cambios de RS (alrededor de 90\%), la diferencia que se observa cada año se atribuye a la condición de saturación atmosférica que, evaluada por medio del DPV, está ausente de la Ec. 1. Para completar los resultados que muestra la Tabla 3 para los años 2007 y 2011, en la Figura 9 se presentan los valores medios mensuales de DPV durante el ciclo de papa para el conjunto de años analizados.

Los DPV media de 2007 y 2009 corresponden al menor y mayor valor del conjunto, respectivamente, lo que explica la marcada diferencia entre la productividad que estiman ambos procedimientos en dichos años. Excepto en 2007, el rendimiento potencial determinado con el modelo que utiliza $w^{D}$ resulta menor al de $e$, de modo que, además de la diferencia que el DPV presenta cada año en el cinturón verde de Córdoba durante el ciclo de papa, no parecen representar alternativas de cálculo que tengan un carácter complementario.

\section{Productividad del agua normalizada con $E T_{o}$}

Evaluando experimentalmente el comporta- miento productivo de diferentes especies de me-

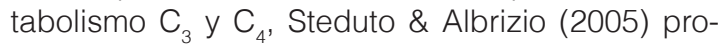
ponen utilizar $\mathrm{ET}_{0}$ a los efectos de normalizar en términos climáticos $w\left(w^{E T o}\right)$ y sostienen que, de esta forma, el método resulta más conservativo o generalizado para estimar el rendimiento potencial de los cultivos (Steduto et al., 2007).

No existe información en la región central de la Argentina respecto a valores de rendimiento potencial estimados a partir del concepto de productividad o eficiencia en el uso del agua. No obstante, evaluaciones realizadas en el cinturón verde de Córdoba sugieren que el rango entre 13 y $18 \mathrm{~g} \mathrm{~m}^{-2}$ utilizado por defecto en el modelo AquaCrop para estimar el rendimiento de las especies $\mathrm{C}_{3}$, estuvo ligeramente por debajo del empleado a los efectos de representar la variabilidad espacial de un lote comercial de papa (de la Casa et al., 2013).

Aplicando este procedimiento de normalización a la información meteorológica del cinturón verde de Córdoba, se obtuvieron los rendimientos potenciales de papa que la Figura 10 presenta respecto a los valores estimados a partir de e para el conjunto de años y fechas de plantación analizadas. El análisis de estos resultados muestra, en primer lugar, que no existe una diferencia significativa $(P>$ $0,05)$ entre los valores medios de ambos procedi- 


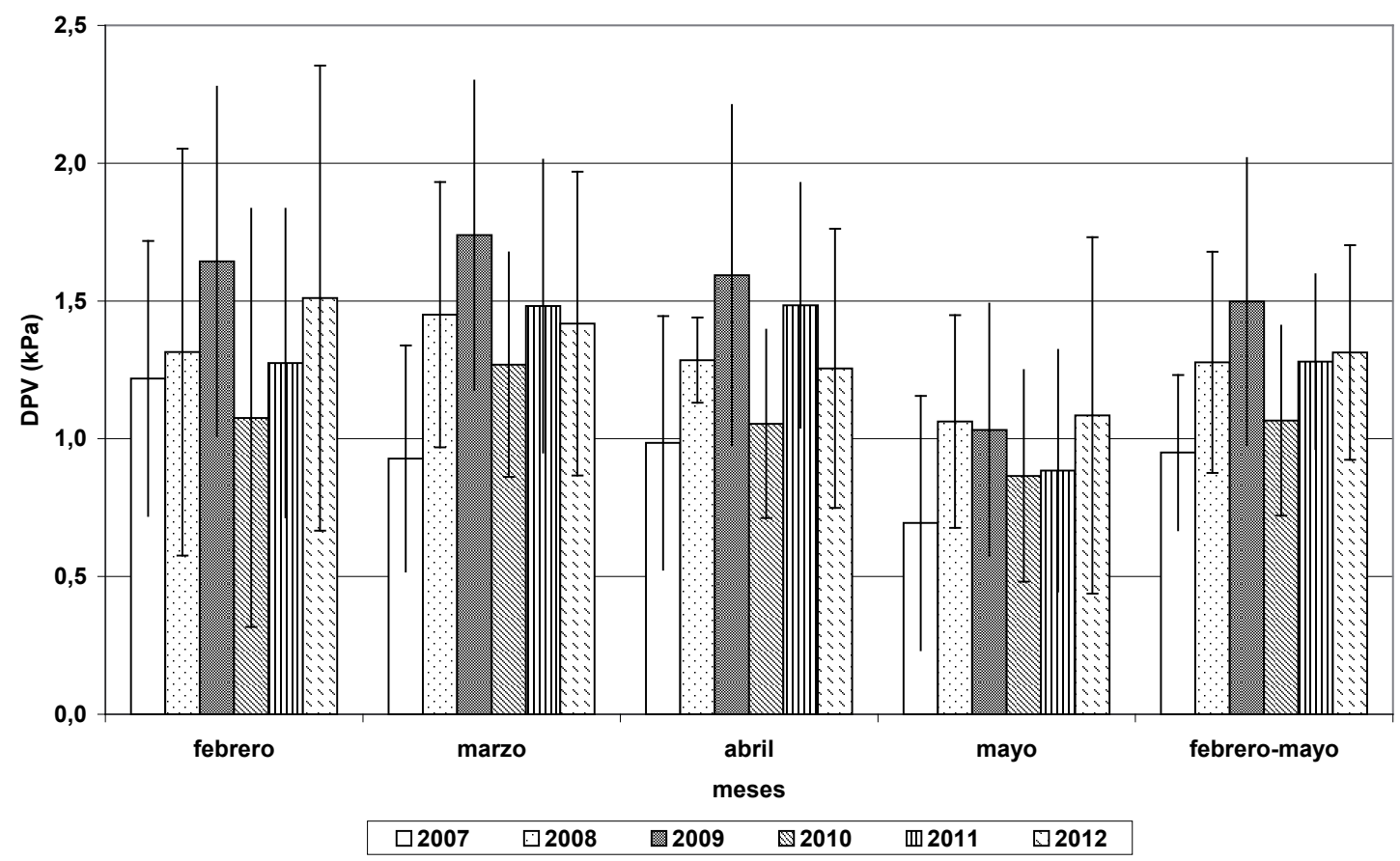

Figura 9. Déficit de presión de vapor (DPV) medio mensual durante el ciclo de papa entre los años 2007 y 2012 en Córdoba, Argentina. La barra sobre el valor medio corresponde a + /- el desvío estándar.

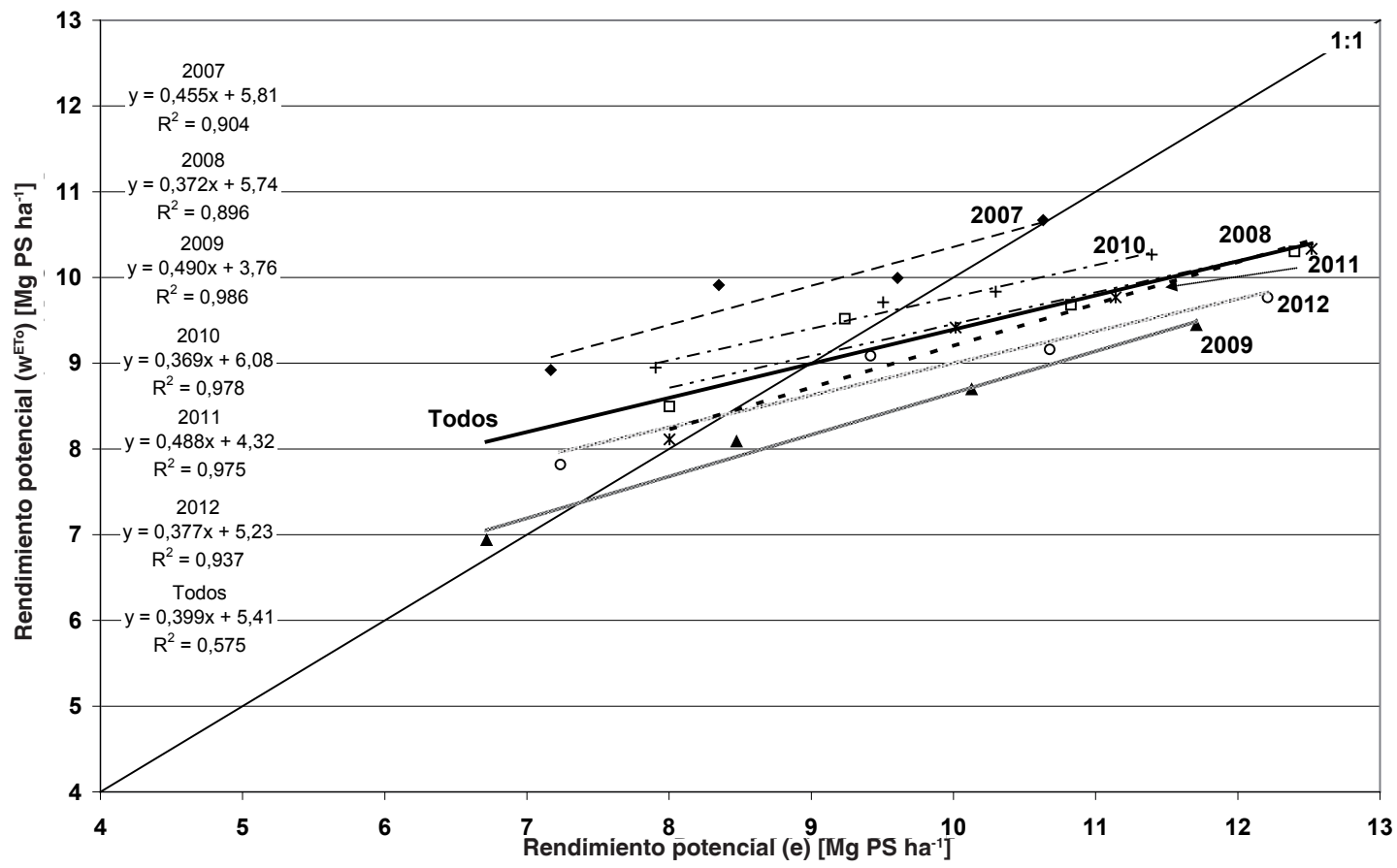

Figura 10. Relación entre los valores de rendimiento potencial obtenidos usando la eficiencia en el uso de la radiación (e) y la eficiencia en el uso del agua normalizada con la evapotranspiración de referencia (wETo), correspondientes a distintas fechas de plantación para cada año entre 2007 y 2012 en Córdoba, Argentina. 
mientos. Además, la normalización del clima a partir de $\mathrm{ET}_{0}$ reduce notablemente la dispersión que se produce al emplear el DPV (Figura 8) y, de esta forma, hay una tendencia mayor a obtener resultados más similares entre e y $w$. El rendimiento potencial que se obtiene a partir de $w^{E T o}$, siendo ligeramente inferior al que produce $e$, reduce notablemente la variabilidad entre años y fechas de siembra ( $C V=9,7 \%)$. De todos modos, cuando se considera el efecto conjunto de la fecha de plantación y del ciclo de producción, el ajuste lineal tanto en la Figuras 8 como en la Figura 10 presenta coeficientes de regresión distintos de 1 para la pendiente y de 0 para la ordenada, indicando un comportamiento particular en cada año. Además, en la Figura 10 persisten diferencias más notables en 2007 y 2009 que demanda la obtención de evidencia experimental a los efectos de convalidar la aptitud de un método en particular.

A partir de un conjunto de datos de rendimiento comercial de papa tardía relevados en el cinturón verde de Córdoba entre 2007 y 2012 (de la Casa et al., 2011, 2013), la Tabla 5 muestra la productividad observada y estimada con los procedimientos destinados a calcular el rendimiento potencial. La relación entre ambos permite establecer una medida de la brecha productiva existente que, dependiendo del año, alcanza un porcentaje entre 40 y $76 \%$ respecto al valor potencial cuando se emplea $e$, y entre $47 \%$ y $81 \%$ al utilizar $w$ normalizada con $E T_{0}$. Estos resultados muestran, en ambos casos, que existe una amplia oportunidad para mejorar la producción de papa en la región.

\section{CONCLUSIONES}

El rendimiento potencial del cultivo de papa de ciclo tardío (otoñal) en el cinturón verde de Córdo- ba se determinó a partir de los dos procedimientos de uso más generalizado: la eficiencia en el uso de la radiación (e) y la eficiencia o productividad del agua $(w)$. Los resultados muestran una importante variabilidad de la productividad potencial, asociada con la fecha de plantación y las condiciones meteorológicas propias de cada ciclo de producción, siendo la dispersión más reducida cuando se emplea el procedimiento de la productividad del agua normalizada a partir de $\mathrm{ET}_{\mathrm{o}}\left(w^{\mathrm{ETO}}\right)$.

Cualquiera sea el método utilizado para estimar el rendimiento potencial, es evidente el efecto negativo que produce el atraso de la plantación respecto a la productividad del cultivo de papa de ciclo otoñal en el cinturón verde de Córdoba, a la vez que la tasa de disminución es ligeramente menor cuando se aplica $w$.

La modelación del crecimiento potencial del cultivo de papa a partir de $w$ constituye un procedimiento más robusto que cuando las estimaciones se basan en $e$, con valores de $w$ que tienen mayor capacidad de extrapolación, siempre que la normalización de la condición climática se realice a través de la evapotranspiración de referencia $\left(E_{0}\right)$ en lugar de utilizar el déficit de presión de vapor (DPV).

\section{BIBLIOGRAFÍA}

Albrizio, R. and P. Steduto, 2005. Resource use efficiency of fieldgrown sunflower, sorghum, wheat and chickpea. I. Radiation use efficiency. Agric. For. Meteorol. 130:254-268.

Allen, R.G.; L.S. Pereira; D. Raes and M. Smith, 1998. Crop evapotranspiration. Guidelines for computing crop water requirements (Irrigation and drainage paper 56). Food and Agriculture Organization, Rome.

Allen, R.G. and L.S. Pereira, 2009. Estimating crop

Tabla 5. Rendimiento observado (RO), con su desvío estándar (DE) y coeficiente de variación (CV), obtenidos en el cinturón verde de Córdoba, Argentina, para los ciclos de papa tardía entre 2007 y 2012. Para cada fecha de plantación se presentan los valores de rendimiento potencial (RP) obtenidos a partir de la eficiencia en el uso de la radiación (e) y de la productividad del agua normalizada a partir de ETo $\left(w^{E T o}\right)$.

\begin{tabular}{cccccccccc}
\hline \multirow{2}{*}{ Año } & \multirow{2}{*}{$\begin{array}{c}\text { Fecha de } \\
\text { plantación }\end{array}$} & $\mathrm{n}$ & \multicolumn{2}{c}{$\mathrm{RO}$} & $\mathrm{DE}$ & $\mathrm{CV}$ & \multicolumn{2}{c}{$\mathrm{RP}(e)$} & \multicolumn{2}{c}{$\mathrm{RP}\left(W^{\text {ETo }}\right)$} \\
\cline { 4 - 10 } & & \multicolumn{2}{c}{$\left(\right.$ Mg PS ha- $\left.^{-1}\right)$} & $(\%)$ & $\left(\mathrm{Mg} \mathrm{PS} \mathrm{ha}^{-1}\right)$ & $\mathrm{RO} \mathrm{RP}^{-1}$ & $\left(\mathrm{Mg} \mathrm{PS} \mathrm{ha-1}^{-1}\right)$ & $\mathrm{RO} \mathrm{RP}^{-1}$ \\
\hline 2007 & $19 / 02 / 2007$ & 29 & 6,44 & 1,1 & 17,1 & 8,48 & 0,76 & 10,06 & 0,64 \\
2008 & $12 / 02 / 2008$ & 8 & 7,84 & 1,5 & 19,1 & 10,51 & 0,75 & 9,65 & 0,81 \\
2009 & $09 / 02 / 2009$ & 16 & 4,12 & 1,0 & 24,3 & 10,29 & 0,40 & 8,77 & 0,47 \\
2010 & $16 / 02 / 2010$ & 52 & 5,65 & 0,8 & 14,2 & 9,82 & 0,58 & 9,76 & 0,58 \\
2011 & $12 / 02 / 2011$ & 28 & 5,32 & 0,9 & 16,9 & 10,91 & 0,49 & 9,70 & 0,55 \\
2012 & $14 / 02 / 2012$ & 22 & 5,74 & 0,9 & 15,7 & 10,17 & 0,56 & 9,13 & 0,63 \\
\hline
\end{tabular}


coefficients from fraction of ground cover and height. Irrigation Science 28:17-34.

Azam-Ali, S.N.; A. Sesay; S.K. Karikari; F.J. Massawe; J. Aguilar-Manjarrez; M. Bannayan and K.J. Hampson, 2001. Assessing the potential of an underutilized cropa case study using a bambara groundnut. Exp. Agr. 37:433-472.

Brisson, N.; C. Gary; E. Justes; R. Roche; B. Mary; D. Ripoche; D. Zimmer; J. Sierra; P. Bertuzzi; P. Burger; F. Bussière; Y.M. Cabidoche; P. Cellier; P. Debaeke; J.P. Gaudillère; C. Hénault; F. Maraux; B. Seguin and H. Sinoquet, 2003. An overview of the crop model STICS. Eur. J. Agron. 18:309-332.

Caldiz, D.O.; F.J. Gaspari; A.J. Haverkort and P.C. Struik, 2001. Agro-ecological zoning and potential yield of single or double cropping of potato in Argentina. Agric. For. Meteorol. 109:311-320.

Cassman, K.G.; A. Dobermann; D.T. Walters and H.S. Yang, 2003. Meeting cereal demand while protecting natural resources and improving environmental quality. Ann. Rev. Environ. Resour. 28:315-358.

de la Casa, A.; Á. Rodríguez y G. Ovando, 1999. Factibilidad del cultivo de papa de secano en la región central de la provincia de Córdoba (Argentina) basada en la evaluación agroclimática del riesgo de sequía. Agriscientia, XVI:55-62.

de la Casa, A.; G. Ovando y Á. Rodríguez, 2003. Estimación de la radiación solar global en la provincia de Córdoba, Argentina, y su empleo en un modelo de rendimiento potencial de papa. Revista de Investigaciones Agropecuarias (INTA), 32(2):45-62.

de la Casa, A. y O.B. Nasello, 2006. Comparación de registros meteorológicos de tres estaciones de la ciudad de Córdoba para extrapolarlos con fines agroclimáticos. Anales AFA 18:295-299.

de la Casa, A.; G. Ovando; L. Bressanini; J. Martínez y Á. Rodríguez, 2011. Eficiencia en el uso de la radiación en papa estimada a partir de la cobertura del follaje. Agriscientia, XXVIII(1):21-30.

de la Casa, A.; G. Ovando; L. Bressanini; J. Martínez y Á. Rodríguez, 2012. Predicción de la variabilidad del rendimiento de papa a partir de la cobertura del follaje. Agriscientia, XXIX(1):15-24.

de la Casa, A.; G. Ovando; L. Bressanini and J. Martínez, 2013. Aquacrop model calibration in potato and its use to estimate yield variability under field conditions. Atmospheric and Climate Sciences, 2013(3):397-407.

Evans, L.T., 1993. Crop evolution, adaptation and yield. Cambridge University Press, Cambridge, UK.

Glenn, E.P.; C.M.U. Neale; D.J. Hunsaker and P.L. Nagler, 2011.Vegetation index-based crop coefficients to estimate evapotranspiration by remote sensing in agricultural and natural ecosystems. Hydrol. Process.
26:4050-4060.

Grassini, P.; H. Yang and K.G. Cassman, 2009. Limits to maize productivity in Western Corn-Belt: A simulation analysis for fully irrigated and rainfed conditions. Agric. For. Meteorol. 149:1254-1265.

Grassini, P.; J. Thorburn; C. Burr and K.G. Cassman, 2011. High-yield irrigated maize in the Western US Corn Belt: I. On-farm yield, yield potential, and impact of agronomic practices. Field Crops Research 120(1):142-150.

Griffin, T.S.; B.S. Johnson and J.T. Ritchie, 1995. A simulation model for potato growth and development: Substor-potato Version 2.0. IBSNAT Research Report Series 02. Dept. of Agronomy and Soil Science.

Haverkort, A.J., 1990. Ecology of potato cropping systems in relation to latitude and altitude. Agric. Syst., 32:251-272.

Hoogenboom, G.J.; W. Jones and K.J. Boote, 1992. Modeling growth, development and yield of grain legumes using SOYGRO, PNUTGRO, and BEANGRO: A Review. Transactions of the ASAE 35.

Jones, C.A. and J.R. Kiniry. 1986. CERES-Maize: A Simulation Model of Maize Growth and Development. Texas A\&M University Press, College Station, Texas.

Jones, C.A.; P.T. Dyke; J.R. Williams; J.R. Kiniry; C.A. Benson and R.H. Griggs, 1991. EPIC: an operational model for evaluation of agricultural sustainability. Agric. Syst. 37:341-350.

Kadaja, J. and H. Tooming, 2004. Potato production model based on principle of maximum plant productivity. Agric. For. Meteorol. 127:17-33.

Khan A.A.; M.S Jilani; M.Q. Khan and M.Zubair, 2011. Effect of seasonal variation on tuber bulking rate of potato. The Journal of Animal \& Plant Sciences, 21(1): 31-37.

Kooman, P.L. and A.J. Haverkort, 1995. Modelling development and growth of the potato crop influenced by temperature and daylength: LINTUL-POTATO. In: Haverkort, A.J., Mac Kerron, D.K.L. (Eds.), Potato Ecology and Modelling of Crops Under Conditions Limiting Growth. Kluwer Academic Publishers, Dordrecht, pp. 41-60.

Kooman, P.L.; M. Fahem; P. Tegera and A.J. Haverkort, 1996a. Effects of climate on different potato genotypes. 1. Radiation interception, total and tuber dry matter production. Eur. J. Agron. 5:193-205.

Kooman, P.L.; M. Fahem; P. Tegera and A.J. Haverkort, 1996b. Effects of climate on different potato genotypes. 2. Dry matter allocation and duration of the growth cycle. Eur. J. Agron. 5:207-217.

Monteith, J.L., 1977. Climate and the efficiency of crop production in Britain. Philos T R Soc Lond B 281:277294.

Mosciaro, M., 2004. Caracterización de la producción y 
comercialización de papa en Argentina. Área de Economía y Sociología Rural. EEA-INTA Balcarce. 13 pp.

Pereira, A.B.; N.A. Villa Nova; V.J. Ramos; A.R. Pereira, 2008. Potato potential yield based on climatic elements and cultivar characteristics. Bragantia 67(2):327-334.

Raes, D., 2009. The ETo Calculator. Reference Manual 3.1. Food and Agriculture Organization of the United Nations (FAO), Land and Water Division. 37 pp.

Raes, D.; P. Steduto; T.C. Hsiao and E. Fereres, 2009. AquaCrop - The FAO crop model to simulate yield response to water. Reference Manual. Chapter 3: Calculation procedures. Food and Agriculture Organization of the United Nations (FAO). 79 pp.

Sinclair, T.R. and R.C. Muchow, 1999. Radiation use efficiency. Adv. Agron. 65:215-265.

Stanhill, G., 1986. Water use efficiency. Adv. Agron. 39:53-85.

Steduto, P. and R. Albrizio, 2005. Resource-use efficiency of fieldgrown sunflower, sorghum, wheat and chickpea. II Water use efficiency and comparison with radiation use efficiency. Agric. For. Meteorol. 130:269281.

Steduto, P., T.C. Hsiao and E. Fereres, 2007. On the conservative behavior of biomass water productivity. Irrig. Sci. 25:189-207.

Steduto, P., T.C. Hsiao, D. Raes and E. Fereres, 2009. AquaCrop-The FAO crop model for predicting yield response to water: I. Concepts and underlying principles. Agron. J. 101:426-437.

Stöckle, C.O. and R.L Nelson, 2000. Cropsyst User's manual (Version 3.0). Biological Systems Engineering
Dept., Washington State University, Pullman, WA.

Stöckle, C.O.; M. Donatelli and R. Nelson, 2003. CropSyst, a cropping systems simulation model. Eur J Agron 18:289-307.

Stol, W.; G.H.J. de Koning; P.L. Kooman; A.J. Haverkort, H. van Keulen and F.W.T. Penning de Vries, 1991. Agro-ecological characterization for potato production. CABO-DLO Report 155, 53 pp.

Tanner, C.B. and T.R. Sinclair, 1983. Efficient water use in crop production: research or re-search? In: Taylor, H.M.; Jordan, W.A. and T.R. Sinclair (eds). Limitations to efficient water use in crop production. ASA, Madison, pp 1-27.

van Keulen, H. and W. Stol, 1995. Agro-ecological zonation for potato production. In: Haverkort, A.J., MacKerron, D.K.L. (Eds.), Potato Ecology and Modelling of Crops Under Conditions Limiting Growth. Kluwer Academic Publishers, Dordrecht, pp. 357-372.

van Ittersum, M.K. and R. Rabbinge, 1997. Concepts in production ecology for analysis and quantification of agricultural input-output combinations. Field Crops Res. 52:197-208.

Worthington, C.M. and C.M. Hutchinson, 2006. Yeild and quality of "Atlantic" and "Harley Blackwell" potatoes as affected by multiple planting dates, nitrogen rates and growing degree days. Proc. Fla. State. Hort. Soc. 119:275-278.

Yang, H.S.; A. Dobermann; J.L. Lindquist; D.T. Walters; T.J. Arkebauer and K.G. Cassman, 2004. Hybrid-Maize: a maize simulation model that combines two crop modeling approaches. Field Crops Res. 87:131-154. 